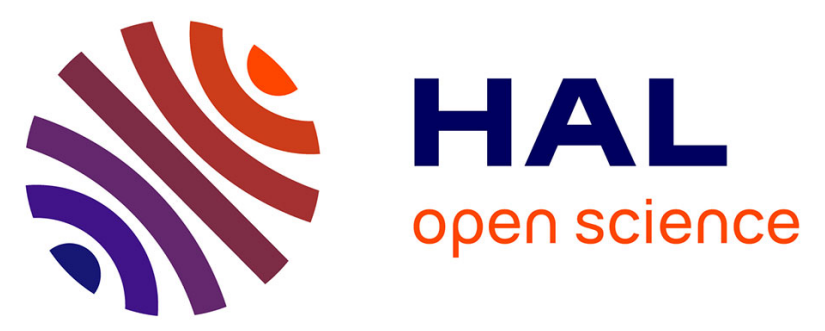

\title{
Systemic growth of Leptosphaeria maculans from cotyledons to hypocotyls in oilseed rape: influence of number of infection sites, competitive growth and host polygenic resistance
}

Renaud Travadon, Bruno B. Marquer, Arnaud Ribule, Ivan I. Sache, Jean-Pierre Masson, Hortense H. Brun, Régine Delourme, Lydia Bousset

\section{To cite this version:}

Renaud Travadon, Bruno B. Marquer, Arnaud Ribule, Ivan I. Sache, Jean-Pierre Masson, et al.. Systemic growth of Leptosphaeria maculans from cotyledons to hypocotyls in oilseed rape: influence of number of infection sites, competitive growth and host polygenic resistance. Plant Pathology, 2009, 58 (3), pp.461-469. 10.1111/j.1365-3059.2008.02014.x . hal-02661250

\section{HAL Id: hal-02661250 \\ https://hal.inrae.fr/hal-02661250}

Submitted on 30 May 2020

HAL is a multi-disciplinary open access archive for the deposit and dissemination of scientific research documents, whether they are published or not. The documents may come from teaching and research institutions in France or abroad, or from public or private research centers.
L'archive ouverte pluridisciplinaire HAL, est destinée au dépôt et à la diffusion de documents scientifiques de niveau recherche, publiés ou non, émanant des établissements d'enseignement et de recherche français ou étrangers, des laboratoires publics ou privés.

$$
\text { Copyright }
$$


Systemic growth of Leptosphaeria maculans from cotyledons to hypocotyls in oilseed rape: influence of number of infection sites, competitive growth and host polygenic resistance

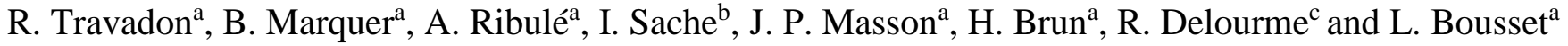

${ }^{a}$ INRA, Agrocampus Rennes, Univ. Rennes 1, UMR1099 BiO3P (Biology of Organisms and Populations applied to Plant Protection), F-35653 Le Rheu;

' INRA, AgroParisTech, UMR1290 BIOGER - CPP, F-78850 Thiverval Grignon; and

'INRA, Agrocampus Rennes, Univ.Rennes 1, UMR118 APBV (Plant Genetics and Biotechnologies), F-35653

Le Rheu, France

\begin{abstract}
The influence of competitive effects between two isolates, of the number of infection sites on cotyledons and of host polygenic resistance on the systemic growth of Leptosphaeria maculans, the cause of phoma stem canker in oilseed rape (Brassica napus), were investigated. Controlled-condition experiments were conducted with two oilseed rape doubled haploid lines, one susceptible and the other with a high level of polygenic resistance, inoculated via wounded cotyledons with conidial suspensions obtained from two isolates. Expression of cankers in plants was enhanced by exposing inoculated plants to low temperature $\left(6^{\circ} \mathrm{C}\right)$ followed by warm temperature $\left(20^{\circ} \mathrm{C}\right)$. The fungus was detected by PCR amplifications of three minisatellite markers in all stems with visible canker symptoms and also in the stems of 14 of the 59 plants without visible cankers on the hypocotyls. Disease severity increased with the number of infection sites on cotyledons: in one of the three replicate experiments, the mean external necrosis length on the hypocotyl ranged from 6.47 to $35.3 \mathrm{~mm}$ for one and eight infections sites on cotyledons, respectively. The probability of an isolate reaching the hypocotyl from inoculated cotyledons decreased with increasing competing inoculum load on cotyledons: for instance, for isolate A290v it decreased from 1 when inoculated alone to $0 \cdot 28$ when coinoculated with six drops of competing isolate P27d. Polygenic resistance significantly reduced disease incidence and severity. For instance, in one of the three replicate experiments, disease incidence ranged from more than $74 \%$ in susceptible plants to $16 \%$ in resistant ones, while mean external necrosis length was up to 35.3 and $6.5 \mathrm{~mm}$ on susceptible and on resistant plants, respectively. This study offers new possibilities for assessing levels of polygenic resistance to stem canker in B. napus and studying the aggressiveness of $L$. maculans isolates.
\end{abstract}

Keywords: Brassica napus, competitive effects, Phoma lingam, phoma stem canker

\title{
Introduction
}

Phoma stem canker (blackleg), affecting Brassica crops worldwide, is the most damaging disease of oilseed rape (Brassica napus), both on winter and spring cultivars. The disease is of major economic importance in Europe, North America and Australia (West et al., 2001; Fitt et al., 2006).

Damaging phoma stem canker is caused by the heterothallic fungus Leptosphaeria maculans (anamorph Phoma lingam). Epidemics are generally initiated by airborne ascospores released from pseudothecia formed on infected oilseed rape stubble remaining from the previous growing season (Hall, 1992). Ascospores first adhere to seedling cotyledons and leaves and then germinate in humid conditions, producing hyphae that cause infection via stomatal pores or wounds. After the incubation period, leaf lesions called phoma leaf spots appear. Following initial leaf infection, mycelial hyphae colonize the lamina mesophyll, then grow down the petiole and stem through xylem vessels or between cells of the xylem parenchyma and cortex in a biotrophic, systemic and visually symptomless way, eventually causing stem cankers (Hammond et al., 1985).

Management of phoma stem canker primarily relies on the cultivation of resistant material (Aubertot et al., 2006b). Resistance to L. maculans in B. napus lines and cultivars is of two kinds. First, race-specific resistance is effective at both the seedling and adult plant stages. Controlled by major genes, this type of resistance is based on gene-for-gene interaction between avirulence genes in L. maculans and matching resistance genes in B. napus (Ansan-Melayah et al, 1995, 1998). Secondly, quantitative and, to date, not racespecific, resistance is polygenic [mediated by quantitative trait loci (QTLs)] and expressed at the adult-plant 
stage. Quantitative resistance in B. napus does not reduce either leaf or cotyledon infection, but such resistant cultivars develop less severe disease before harvest than susceptible cultivars (Delourme et al., 2006).

Two major resistance genes were overcome in France ( $R \operatorname{lml} 1$ introduced into several winter cultivars) and Australia (resistance from B. rapa var. sylvestris introduced into cv. Surpass 400) within 3 years of their commercial deployment (Li \& Sivasithamparam, 2003; Rouxel et al., 2003; Sprague et al., 2006). Similarly, Rlm6, not currently present in commercial cultivars, was overcome over 3 years in experimental conditions (Brun et al., 2000). Loss of effectiveness of a major resistance gene corresponds to a rapid increase in the frequency of the matching virulence in pathogen populations, which illustrates the high evolutionary potential of L. maculans. The adaptation of pathogen populations first requires the appearance of virulent isolates and secondly the transmission of virulence to offspring so that this trait can become a selective advantage. Virulence transmission to offspring in L. maculans populations occurs either through asexual multiplication or sexual reproduction. Mycelium survives asexually on oilseed rape stem debris (stubble) during intercropping and finally produces pycnidia (Petrie \& Lewis, 1985; Baird et al., 1999). As splash-dispersed conidia of L. maculans travel short distances (Travadon et al., 2007), virulence transmission requires the cultivation of oilseed rape in the same field as stubble from previous oilseed rape crops. Alternatively, sexual reproduction results in pseudothecia formation and ascospore release. Wind-dispersed over several kilometres (McGee, 1977), ascospores may encounter a resistant host grown in distant fields.

A prerequisite to sexual reproduction is the mating of compatible isolates in the same oilseed rape stem. Direct colonization of wounded stems was postulated from a field study (Xi et al., 1991). More generally, systemic growth of $L$. maculans from an infected leaf or cotyledon to the stem is a necessary step before sexual reproduction, and is thus essential for large-scale adaptation of pathogen populations to major resistance genes in B. napus. This systemic, biotrophic stage could act as a filter for $L$. maculans individuals present on leaves and cotyledons if only some of them succeeded in stem colonization. Stem colonization success might be moderated by leaf infection densities and by host polygenic resistance. Competition between L. maculans isolates, defined as the negative effects that one organism has upon another by consuming, or controlling access to, a resource that is limited in availability (Begon et al., 2006), could alter the capacity of a given isolate to reach the stem and further to mate sexually. Among coexisting organisms, the effects of competition on reproductive output increase with rising population density (Begon et al., 2006); thus the fitness of each strain, i.e. the per-individual contribution to the next generation's gene pool (Pringle \& Taylor, 2002), will depend on the absolute densities of all strains in mixed populations (Newton et al., 1997). Therefore, the dynamics of systemic growth might be an essential component of the relative fitness of $L$. maculans individuals.

Previous studies on the systemic pathway of L. maculans were conducted in the field (Hammond \& Lewis, 1986; Xi et al., 1991; Schmit et al., 2002), while experiments in controlled conditions focused on mycelial growth, either in the leaf lamina (Hammond \& Lewis, 1987), or from the leaf lamina to the petiole (Hammond et al., 1985; Xi et al., 1991; Huang et al., 2007, 2009), using either petiole inoculation (Newman, 1984; Kutcher et al., 1993) or direct stem inoculation (Nathaniels \& Taylor, 1983). As attempts to use the published methods for allowing the development of stem canker after cotyledon inoculation (Bansal et al., 1994; Johnson \& Lewis, 1994) were not successful under the experimental conditions here, a prerequisite for this study was to set up and validate a reliable inoculation method in controlled conditions. Using that method, the aims of the present work were (i) to evaluate the effect of number of infection sites on the cotyledon on stem canker incidence and severity, (ii) to assess the effect of polygenic resistance on the systemic growth of the fungus, and (iii) to assess the probability of stem colonization by one isolate inoculated on the cotyledon or co-inoculated with an increasing load of a competing isolate.

\section{Materials and methods}

\section{Inoculum and plant material}

Two isolates of L. maculans, A290v genotype (Mat1-2, AvrLm1, avrLm2, avrLm3, avrLm4, AvrLm5, AvrLm6, AvrLm7, AvrLm8, avrLm9, AvrLm10) (Somda et al., 1999) and P27d genotype (Mat1-2, AvrLm1, avrLm2, avrLm3, avrLm4, AvrLm5, AvrLm6, AvrLm7, avrLm9, AvrLm10), were obtained from single ascospores isolated from diseased B. napus stubble collected in Le Rheu (France) according to the method described by 
Smith \& Sutton (1964). Inoculum, consisting of suspensions of $10^{7}$ conidia $\mathrm{mL}^{-1}$, was obtained as described by De March et al. (1986). Experiments were conducted with two doubled haploid lines of B. napus derived

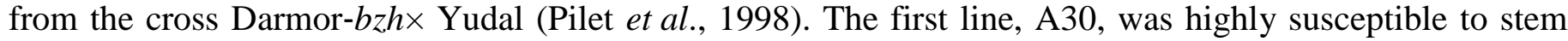
canker, whereas adult plants from the second line, $\mathrm{C} 119$, had a high level of field polygenic resistance to stem canker. Resistance levels in both lines were assessed in field trials for two seasons; disease ratings at the adult plant stage based on a mean disease index (Pilet et al., 1998) were 5.7-9 and 1.2-2.9 for lines A30 and C119, respectively (Roussel, 1999). Both isolates induced very susceptible and equal reactions on both lines at the cotyledon stage (Roussel, 1999). The genetic basis of quantitative resistance in the progeny of the Darmor$b z h \times$ Yudal cross includes many QTLs implied in resistance expression to L. maculans (Pilet et al., 1998).

\section{Development of stem canker assessment methodology}

After pregermination, 192 seeds of the susceptible line A30 were sown in Fertiss ${ }^{\circledR}$ medium (1:1:1 blond sphagnum peat/perlite/vermiculite) in $4-\times 6-\mathrm{cm}$ propagation plugs. Seedlings were grown in a glasshouse at $20^{\circ} \mathrm{C}$ under a 16-h photoperiod for 10 days. The cotyledons were then slightly wounded in the centre of each lobe with a needle, and a $10-\mu \mathrm{L}$ drop of a conidial suspension $\left(10^{7}\right.$ conidia $\left.\mathrm{mL}^{-1}\right)$ was deposited on each wound. Accordingly, each plant was inoculated with four drops. Plants treated with distilled water instead of spore suspension served as controls. A plastic cover was placed over the inoculated plants to give $100 \%$ relative humidity $(\mathrm{RH})$. The inoculated plants were incubated in the dark at $20 \pm 2^{\circ} \mathrm{C}$ for $72 \mathrm{~h}$ in a growth chamber and then placed in an atmosphere with $70 \% \mathrm{RH}$ (without a plastic cover) with a 16-h photoperiod. Cotyledon senescence was delayed by leaving only the last emerged young leaf and removing the others during the 14 days after inoculation. Infection efficiency was assessed 14 days and 21 days after inoculation by checking phoma leaf spot appearance. Twenty-one days after inoculation, the 192 plants were split into four groups of 48 plants. These groups were placed in a growth chamber at $6 \pm 1{ }^{\circ} \mathrm{C}$ (low temperature) with a 16-h photoperiod and $60 \% \mathrm{RH}$ for either $0,4,6$ or 8 weeks. Afterwards, plants were placed in a growth chamber at $20 \pm 2{ }^{\circ} \mathrm{C}$ with a 16 -h photoperiod and $60 \% \mathrm{RH}$. Each of the four groups of 48 plants was then split into four subgroups of 12 plants grown for either $0,2,4$ or 6 weeks before each plant was visually checked for external and internal stem cankers. Disease incidence was computed as the proportion of plants with external stem cankers for the 16 different cultural conditions.

Influence of number of infection sites on the cotyledon and of host polygenic resistance on incidence and severity of stem cankers

Plants were inoculated and placed in growth conditions conducive to stem canker development selected from the experiment described above. In each of three repeated experiments (experiments 1-3), 1, 2, 4 or 8 sites per plant were inoculated by placing a single drop of A290v conidial suspension $(10 \mu \mathrm{L})$ over each site. Eighteen plants each of the susceptible line A30 and the polygenic resistant line C119 were tested for each inoculum load per experiment. Control plants (inoculum load 0) were inoculated with distilled water. For inoculum loads 1, 2 (one drop per cotyledon) and 4 (two drops per cotyledon) infection sites were in the centre of cotyledon lobes. For inoculum load 8, there were two infection sites per cotyledon lobe, one near the base of the half-lamina with respect to petiole insertion (lower position), the other $1 \mathrm{~cm}$ higher (upper position) (Fig. 1). Stem canker was assessed by visual observations of external and internal longitudinal sections of hypocotyl, computing disease incidence as the proportion of plants with external stem cankers. Disease severity was assessed as both the length of external stem cankers and the score of internal stem cankers in a cross section on a 1-6 scale (1, healthy plant, no visible lesions; $2,0-25 \%$ discoloured cross-section; $3,>25-$ 50\% discoloured cross-section; 4, > 50-75\% discoloured cross-section; 5, > 75-100\% discoloured section; 6, section without any living tissue) (Aubertot et al., 2006a).

Figure 1 Schematic layout of a Brassica napus cotyledon infected with four inoculum drops of Leptosphaeria maculans. Upper and lower positions for inoculum drops are defined according to the half-lamina (dashed line). 


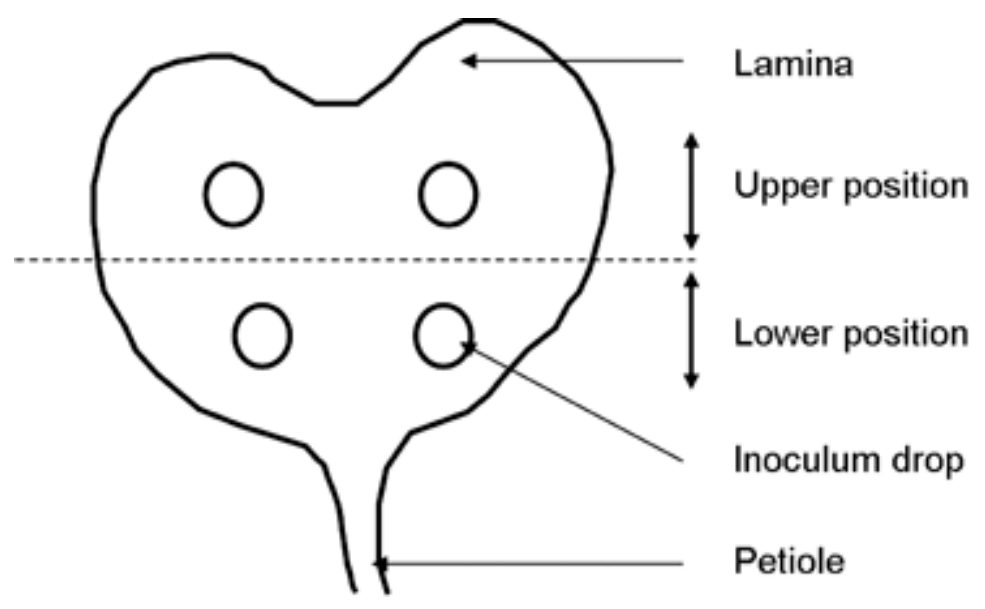

Intraspecific competition

Conidial suspensions of isolates A290v and P27d were inoculated onto cotyledons of the susceptible line A30 as described above. Three replicated experiments consisted each of nine treatments (four ratios for each of the two isolates plus one control inoculated with distilled water) with 12 plants per treatment. The four inoculation ratios were two inoculum drops of one isolate (the tested isolate) either co-inoculated with two drops of water or co-inoculated with two, four or six drops of the second isolate (the competing isolate). Each site was inoculated with a single inoculum drop (e.g. ratio $2 / 6$ had eight infection sites per plant). When more than four drops were inoculated per plant (inoculation ratios 2/4 and 2/6), each isolate inoculation position on the cotyledon (upper or lower, as previously described) was considered (Fig. 1) to assess its effect on hypocotyl colonization success. For fungal detection in the hypocotyl, a sample of 115 plants from the three experiments was tested, consisting of 56 plants that had internal or external stem cankers and 59 plants that were visually symptomless. The 115 plants included 16 plants from experiment 1, 27 plants from experiment 2 and 72 plants from experiment 3 . The 115 plants were randomly selected from the three experiments to balance both the number of plants in each of the nine treatments and the number of plants either with visible symptoms or symptomless. From each plant, three hypocotyl samples of about $2 \times 5 \mathrm{~mm}$ were cut off with a sterile scalpel from the stem cortex to the stem pith. The first sample was cut from the hypocotyl base, the second from the middle of the hypocotyl and the third right underneath the cotyledon insertion point. Genomic DNA was extracted following the standard protocol supplied in the Nucleospin ${ }^{\circledR}$ Plant II kit (Macherey-Nagel) from a mix of the three hypocotyl samples. The presence of $L$. maculans in samples was detected by PCR amplifications of three minisatellite markers: MinLm2 (accession number AJ621801), MinLm6 (accession number AJ621805) and MinLm9 (M.H. Balesdent, INRA-UMR1290, BIOGER-CPP, F-78026 Versailles Cedex, France, personal communication), each marker being polymorphic between the A290v and P27d isolates. The PCR reactions were performed in a 20- $\mu \mathrm{L}$ final volume containing 10-30 ng genomic DNA, 1× PCR buffer, $200 \mu \mathrm{m}$ dNTPs, $1.5 \mathrm{~mm} \mathrm{MgCl}_{2}$, each primer at $0.5 \mu \mathrm{m}$ and $0.25 \mathrm{U}$ GoTaq ${ }^{\circledR}$ DNA Polymerase (Promega). Amplifications were carried out in a G-STORM GS4 thermocycler (GRI Ltd) using one cycle of $94^{\circ} \mathrm{C}$ for $4 \mathrm{~min}$ and 30 cycles of $94^{\circ} \mathrm{C}$ for $30 \mathrm{~s}, 60^{\circ} \mathrm{C}$ for $30 \mathrm{~s}$ and $72^{\circ} \mathrm{C}$ for $1 \mathrm{~min}$, followed by $5 \mathrm{~min}$ of final extension at $72^{\circ} \mathrm{C}$. Amplified DNA products were separated by electrophoresis of $5-\mu \mathrm{L}$ aliquots of PCR mixtures on $2.5 \%$ agarose gels in $1 \times$ TBE buffer and visualized following SYBRSafeTM treatment (Invitrogen) and UV illumination. The sensitivity of PCR reactions to the amount of genomic DNA of each isolate in the PCR mixture was checked. For the three minisatellite markers tested, genomic DNA from both isolates was always amplified when proportions in the PCR mixture varied from $1 \mathrm{ng}$ of one isolate with $25 \mathrm{ng}$ of the other to $15 \mathrm{ng}$ of each isolate.

\section{Statistical analysis}

To test equality of disease incidence between experiments, confidence intervals for incidence differences were calculated as:

$\mathrm{IC}=p_{a}-p_{b} \pm 1.96 \times \sqrt{ }\left[\left(p_{a} \times q_{a} /\left(n_{a}-1\right)\right)+\left(p_{b} \times q_{b} /\left(n_{b}-1\right)\right)\right]$

where $p_{a}$ and $p_{b}$ were disease incidences in each experiment, $q_{a}$ and $q_{b}=1-p, n_{a}$ and $n_{b}$ were the number of samples in each experiment and 1.96 corresponded to the $95 \%$ confidence level. 
Disease severity data were analysed by linear models using the glm procedure of sas (SAS, 2000). A linear model was used to analyse the effect of the experiment, of the number of infection sites, of the line and of their interactions on disease severity (external necrosis length and necrosis score). Multiple comparisons between all pairs of means were performed by the Student-Newman-Keuls method. Linear regression and covariance analysis between internal necrosis length and the other dependent variables were performed with sas.

To assess the effect of the competing inoculum load on the probability of the initial isolate colonizing the hypocotyl and on the probability of the competing isolate colonizing the hypocotyl, generalized linear models were performed using the genmod procedure of sas (SAS, 2000).

\section{Results}

Development of stem canker assessment methodology

Twenty-one days after cotyledon inoculation (21 d.p.i.) no plant had a diseased hypocotyl. Of the plants not exposed to $6^{\circ} \mathrm{C}$ after 21 d.p.i., only a few grown for another 4 or 6 weeks at $20^{\circ} \mathrm{C}$ developed cankers (Fig. 2). In plants exposed to $6^{\circ} \mathrm{C}$ for 4,6 or 8 weeks after 21 d.p.i., disease incidence increased with the number of subsequent weeks at $20^{\circ} \mathrm{C}$. In the longest experiments, all tested plants developed stem cankers. To maximize disease incidence while minimizing the duration of the experiment, a temperature sequence of 4 weeks at $6^{\circ} \mathrm{C}$ followed by 6 weeks at $20^{\circ} \mathrm{C}$ (Fig. 2) was retained as the standard procedure for all the experiments further described in this paper.

Figure 2 Effect of temperature sequence (number of weeks at $6^{\circ} \mathrm{C}$ followed by number of weeks at $20^{\circ} \mathrm{C}$ ) starting 21 days after Leptosphaeria maculans inoculation on the incidence of stem canker on Brassica napus in controlled conditions.

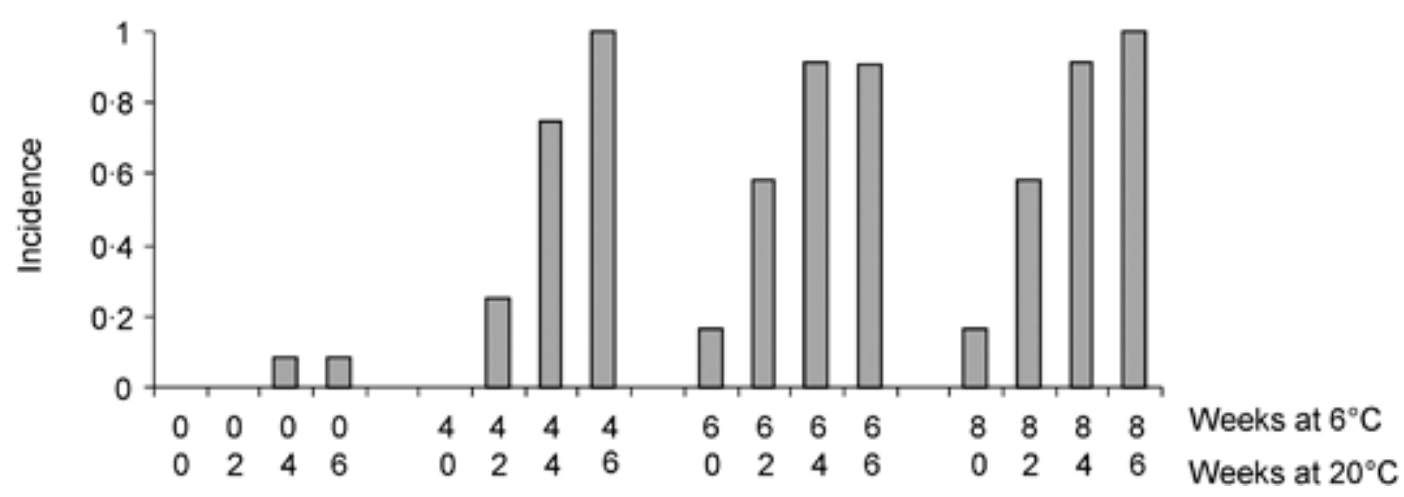

Influence of number of infection sites and of host polygenic resistance on incidence and severity of stem cankers

On the susceptible line A30, the incidence of stem cankers significantly differed between experiments, with incidence in experiment $2>$ experiment 3 (IC $=[0 \cdot 04 ; 0 \cdot 34], P=0 \cdot 05,140$ d.f.), and incidence in experiment $3>$ experiment 1 ( IC $=[0 \cdot 16 ; 0 \cdot 46], P=0 \cdot 05,140$ d.f.) $($ Table 1$)$. On the resistant line $\mathrm{C} 119$, the incidence of stem cankers did not significantly differ between experiments 1 and 2 (IC $=[-0 \cdot 03 ; 0 \cdot 19], P=0 \cdot 05,140$ d.f.), but was significantly higher in experiment 3 (IC $=[0 \cdot 02 ; 0 \cdot 3], P=0 \cdot 05,140$ d.f.) (Table 1$)$.

Table 1. Incidence and severity [mean external necrosis length, mean necrosis score and standard error (SE)] of stem canker caused by Leptosphaeria maculans in Brassica napus lines with (C119) or without (A30) polygenic resistance, in three experiments. Slopes and coefficients of determination of linear models relating internal necrosis length to external necrosis length for each line in each experiment are presented 


\begin{tabular}{|c|c|c|c|c|}
\hline & B. napus line & Exp1 & Exp2 & Exp3 \\
\hline \multirow[t]{2}{*}{ Incidence (\%) } & A30 & $24 \cdot 28$ & $74 \cdot 65$ & $55 \cdot 56$ \\
\hline & C119 & $8 \cdot 57$ & $16 \cdot 67$ & $32 \cdot 56$ \\
\hline \multirow[t]{2}{*}{ Mean external necrosis length (mm) (SE) } & АЗО & $2 \cdot 90(6 \cdot 71)$ & $20 \cdot 31(21 \cdot 46)$ & $11.96(19.25)$ \\
\hline & C119 & $0.66(2.94)$ & $2 \cdot 09(5 \cdot 86)$ & $0.99(4.55)$ \\
\hline \multirow{2}{*}{ Mean necrosis score (SE) } & A30 & $1 \cdot 43(1 \cdot 09)$ & $3 \cdot 16(2 \cdot 20)$ & $2 \cdot 57(2 \cdot 21)$ \\
\hline & C119 & $1 \cdot 14(0 \cdot 63)$ & $1.31(0.92)$ & $1.32(0.69)$ \\
\hline \multirow[t]{2}{*}{ Internal necrosis length $(\mathrm{mm})(\mathrm{SE})^{\mathrm{a}}$} & А30 & $1 \cdot 62(0.81)$ & $1.01(0 \cdot 79)$ & $0.95(0.95)$ \\
\hline & $\mathrm{C} 119$ & $2 \cdot 26(0 \cdot 73)$ & $1.44(0.79)$ & $1 \cdot 21(0 \cdot 63)$ \\
\hline
\end{tabular}

${ }^{a} Y=a+b X$, with $X$ the corresponding external necrosis length (mm) and $b$ the regression slope $\left(R^{2}\right)$.

Severity, either expressed as the external necrosis length or the internal necrosis score of stem cankers, significantly differed between experiments $\left(P\left[F_{(2 ; 467)}>20 \cdot 18\right]<0.0001\right.$ for external necrosis length; and $P$ $\left[F_{(2 ; 467)}>16 \cdot 64\right]<0.0001$ for internal necrosis score $)$, with disease severity in experiment $2>$ experiment 3 $>$ experiment 1 (Table 1$)$.

For each experiment and line, internal necrosis length could be estimated by external necrosis length because a linear model $(Y=a+b X$, with $Y$ the internal necrosis length and $X$ the external necrosis length) linked these two variables. From this, it could be deduced that $(Y-Y m e a n)=b(X-X m e a n)$, with Ymean and Xmean the means of $Y$ and $X$, respectively. For each linear model applied to one line in one experiment, the coefficient of determination $R^{2}$ was higher than 0.63 (Table 1). Covariance analysis revealed that the introduction of the external necrosis length to explain the internal necrosis length was sufficient, while the factor 'number of infection sites' did not improve the explanatory value of the model. Values of slope $b$ greater than 1 indicated that internal necrosis was slightly longer than external necrosis in all but one case.

In each of the three experiments, the polygenic resistance of the $\mathrm{C} 119$ line significantly reduced both disease incidence ( $\mathrm{IC}=[0 \cdot 04 ; 0 \cdot 28], P=0 \cdot 05,140$ d.f. in experiment $1 ; \mathrm{IC}=[0 \cdot 45 ; 0 \cdot 71] P=0 \cdot 05,140$ d.f. in experiment $2 ; \mathrm{IC}=[0.07 ; 0.39], P=0.05,140$ d.f. in experiment 3$)$ (Table 1$)$ and disease severity $\left(P\left[F_{(1 ;}\right.\right.$ 173) $>8.16]<0.005$ for external necrosis length and $P\left[F_{(1 ; 173)}>4.74\right]<0.03$ for internal necrosis score in experiment 1; $P\left[F_{(1 ; 177)}>60.33\right]<0.0001$ for external necrosis length and $P\left[F_{(1 ; 177)}>53 \cdot 88\right]<0 \cdot 0001$ for internal necrosis score in experiment $2 ; P\left[F_{(1 ; 114)}>17 \cdot 28\right]<0.0001$ for external necrosis length and $P\left[F_{(1 ;}\right.$ $\left.{ }_{114)}>16 \cdot 26\right]<0 \cdot 0001$ for internal necrosis score in experiment 3) (Table 1). For instance, in experiment 2, where disease was the most severe, external necrosis length was up to $35.3 \mathrm{~mm}$ on susceptible A30 plants, but only up to $6.5 \mathrm{~mm}$ on resistant $\mathrm{C} 119$ plants (Fig. 3).

Figure 3 Effect of number of Leptosphaeria maculans cotyledon infection sites on external necrosis length and necrosis score for two oilseed rape (Brassica napus) doubled haploid lines (A30: susceptible; C119: with polygenic resistance) in three experiments. Means and standard errors are presented. Means followed by the same letter (upper case: A30; lower case: C119) are not significantly different according to the StudentNewman-Keuls-test $(P<0 \cdot 05)$.

Experiment 1
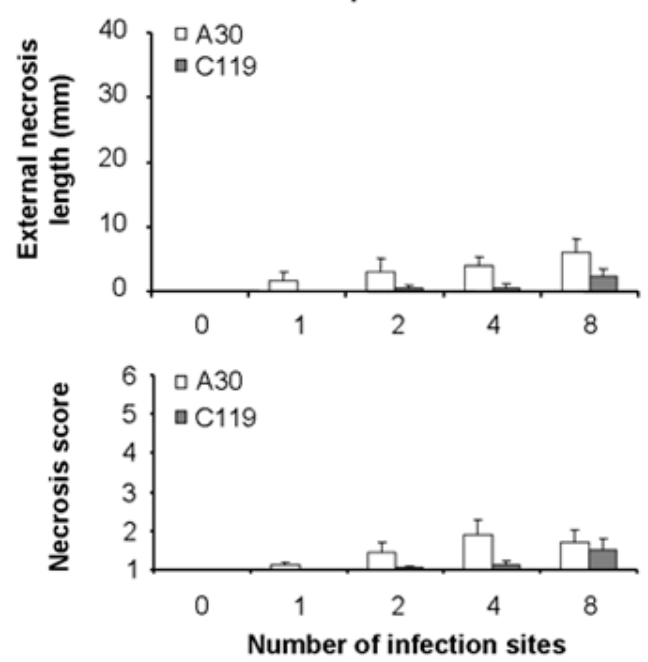

Experiment 2
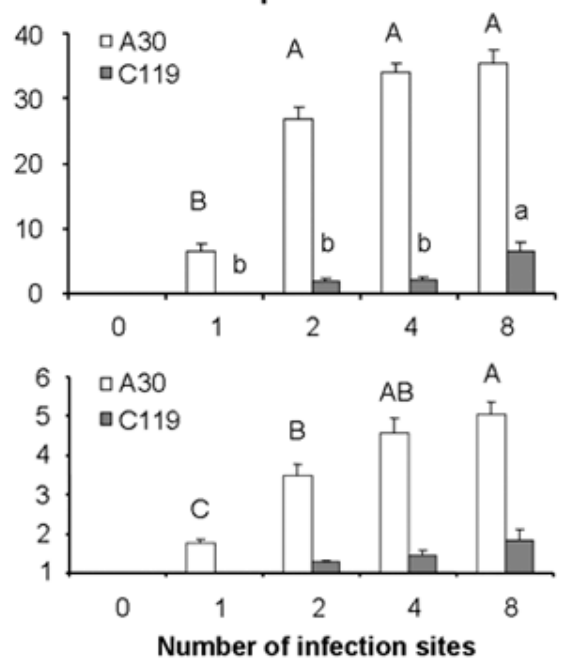

Experiment 3
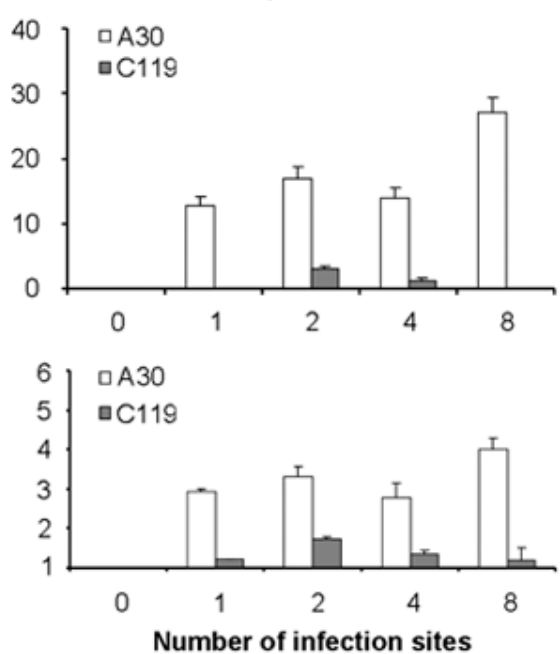
On the susceptible line A30, disease severity (external necrosis length and necrosis score) increased with increasing number of infection sites on cotyledons. For instance, in experiment 2, the mean external necrosis length on hypocotyls ranged from 6.47 to $35.3 \mathrm{~mm}$ for one and eight infections sites on cotyledons, respectively (Fig. 3). The effect of number of infection sites was significant only for experiment $2\left(P\left[F_{(3}\right.\right.$; 67) $>9.68]<0.0001$ for external necrosis length and $P\left[F_{(3 ; 67)}>11.95\right]<0.0001$ for internal necrosis score), where disease severity was higher than in experiments 1 and 3. For instance, maximal external necrosis length on individual plants from line A30 was up to $40 \mathrm{~mm}$ in experiments 2 and 3 , but only up to $8 \mathrm{~mm}$ in experiment 1 (data not shown).

On the resistant line $\mathrm{C} 119$, disease severity increased with increasing number of infection sites on cotyledons only in experiments 1 and 2, the increase being significant for external necrosis length in experiment 2 only $\left(P\left[F_{(3 ; 68)}>3.74\right]<0.01\right)$ (Fig. 3). Results were more ambiguous in experiment 3: external necrosis was visible only for two and four infection sites on cotyledons and their length was not significantly different. Necrosis score was higher than 1 (healthy plant) where no external necrosis was visible, but did not significantly differ among inoculated plants (1.22 and 1.20 for one and eight infection points on cotyledons, respectively). In this experiment, resistant plants had internal necrosis without external necrosis.

\section{Intraspecific competition}

On DNA extracted from hypocotyl samples, L. maculans minisatellite markers were never amplified for control plants inoculated with distilled water, but always amplified for the 56 plants with visible cankers. Both isolates were detected in eight diseased plants which had been inoculated with at least six inoculum drops on cotyledons (Fig. 4).

Figure 4 Probability of hypocotyl colonization by two competing Leptosphaeria maculans isolates (tested isolate, inoculated at two infection sites on cotyledons, or competing isolate) on susceptible Brassica napus plants (doubled haploid line A30) with external hypocotyl necrosis, according to the number of sites infected on cotyledons with the competing isolate.

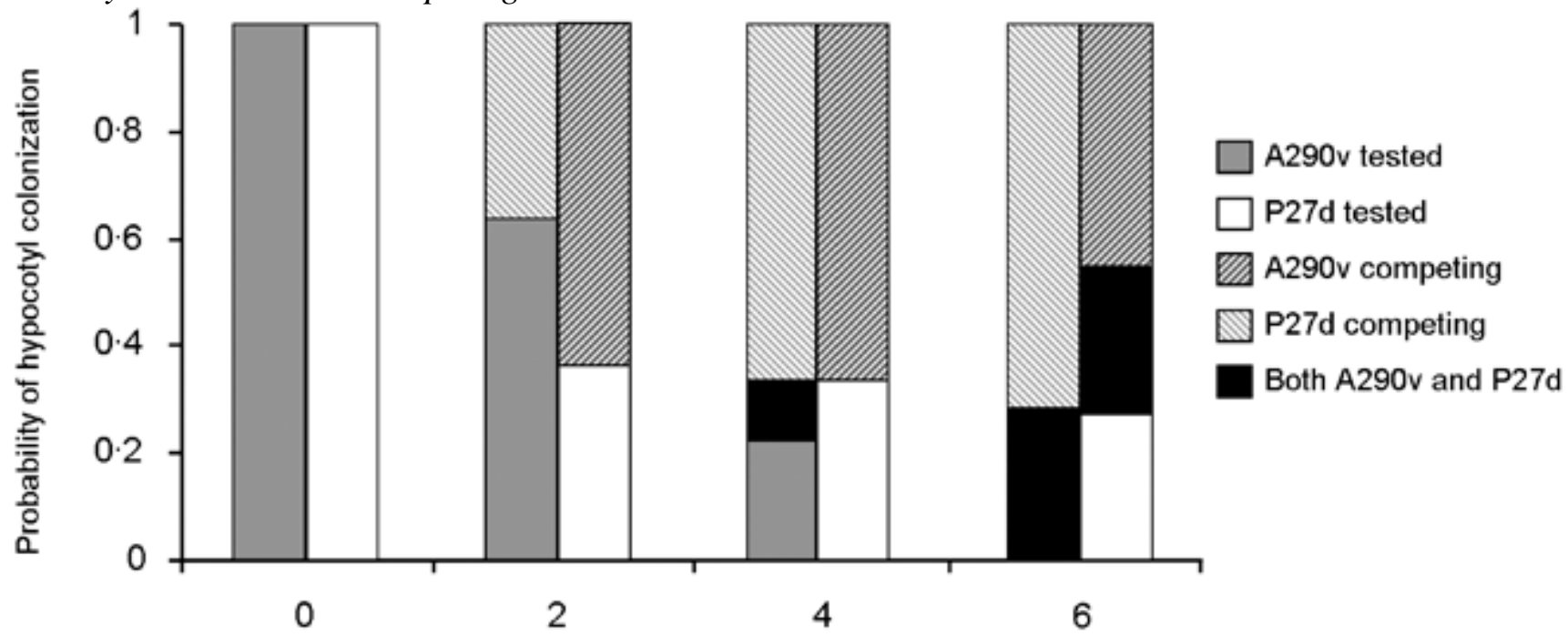

Number of sites infected with competing isolate

When the analysis focused on plants with visibly cankered hypocotyls and when the competing inoculum load 0 was considered, the competing inoculum load had a significant effect on the presence of the tested isolate in the hypocotyl for both cross-inoculations $(P=0 \cdot 004,3$ d.f., for isolate P27d as the competing isolate; $P=$ 0.037, 3 d.f., for isolate A290v as the competing isolate) (Fig. 4). In these plants with cankers, the probability of each isolate colonizing the hypocotyl was equal to 1 without competition (competing inoculum load 0). For isolate A290v, the probability of colonizing the hypocotyl decreased with the increasing competing P27d inoculum load: this probability decreased from 1 when inoculated alone to 0.28 when coinoculated with six drops of competing isolate P27d (Fig. 4). For isolate P27d, results were similar, except the probability of colonizing the hypocotyl was higher for competing A290v inoculum load 6 than for competing A290v 
inoculum loads 2 and 4, because tested isolate P27d was frequently detected with the competing isolate A290v inoculated at six infections sites (Fig. 4).

When the competing inoculum load 0 was excluded from the analysis, the probabilities of each tested isolate colonizing the hypocotyl did not significantly decrease with increasing competing inoculum load. For both isolates the probability of colonizing the hypocotyl increased with their load on cotyledons when used as the competing isolate (Fig. 4), but this effect was significant only for competing isolate P27d $(P=0 \cdot 005,2$ d.f.).

Among the 59 visually symptomless plants tested, detection was successful in 14 plants (23\%), of which 11 plants had been inoculated with at least six inoculum drops on cotyledons. For plants inoculated with at least six or eight inoculum drops on cotyledons, the position of the tested isolate on the cotyledons (lower or upper position) did not affect the probability of colonization of the hypocotyl.

\section{Discussion}

This work provides a reliable methodology to study both the systemic growth of $L$. maculans in its host $B$. napus and the competition between isolates during this growth, requiring the use of low temperature followed by warm temperature to enhance stem canker expression from cotyledon inoculations. This study also highlighted the efficacy of the host carrying polygenic resistance to reduce incidence and severity of stem cankers and revealed that disease severity on hypocotyls increases with the number of infection sites on cotyledons. It further suggests that more infection sites on cotyledons result in a greater probability of reaching the hypocotyl via systemic growth when two isolates are co-inoculated on the same plant.

Expression of cankers in plants was enhanced by exposing inoculated plants to low temperatures (Fig. 2). These growing conditions did not inhibit the systemic growth of the fungus, which has been shown to achieve significant mycelial growth at temperatures as low as $3^{\circ} \mathrm{C}$ (Ndimande, 1976). Furthermore, these conditions simulate the field temperatures experienced by oilseed rape winter cultivars in Western Europe, where seedling development until the rosette stage occurs at the beginning of autumn, when climatic conditions are temperate, followed by cold temperatures during winter inhibiting plant growth. After a 4-week period at $6^{\circ} \mathrm{C}$, plants were returned to a growth chamber at $20 \pm 2^{\circ} \mathrm{C}$ for 6 weeks. Previous studies showed that the transfer of inoculated, symptomless plants to warmer conditions induces very rapid canker development (Brunin \& Lacoste, 1970; Nathaniels \& Taylor, 1983). This observation was confirmed here and results showed that increasing the warm growing period resulted in an increase in disease incidence. In agreement with previous results (Nathaniels \& Taylor, 1983; Xi et al., 1991) the fungus was detected in visually symptomless plants. Results suggest, however, that the development of cankers in symptomless plants may have required a longer time period before assessment.

Disease incidence and severity displayed variability among the three experiments (Table 1). Plants were grown in a glasshouse for 10 days before inoculation; expected variations in temperature, light intensity or relative humidity between experiments could have resulted in differences in plant physiological status at inoculation. All experiments, however, were performed in the same growing chambers, with only 1 week between repeats. A more likely hypothesis involves the conditions during the incubation period following inoculation. Germination of conidia of L. maculans and subsequent infection requires continuous wetness for at least $16 \mathrm{~h}$ (Hall, 1992). High relative humidity was ensured by placing a plastic cover over inoculated plants, but in the absence of a reliable method of assessment of water films on leaf surfaces, the homogeneity of leaf wetness, a prerequisite to homogeneous mycelial colonization of cotyledon lamina, could not be checked. Indeed, interrupted wet periods have major effects on the establishment of fungal leaf diseases (Huber \& Gillespie, 1992), especially L. maculans, in which conidial germination is inhibited by short dry periods (Hall, 1992). Histological studies (Hammond \& Lewis, 1987) described three causes of failure to establish systemic biotrophic infection after inoculation. The first two causes (infection failure before lamina penetration; and fungal restriction within $300 \mu \mathrm{m}$ of the pin-prick margin after lamina penetration) can be rejected here because phoma leaf spots were actually observed 14 and 21 days after inoculation. The third possible cause (host cell necrosis overtaking the hyphal front and growth stopping after lamina colonization) could probably explain the inter-experiment variability in disease incidence and severity; intra-experiment variability was low, as indicated by low standard errors on Fig. 2. To reduce inter-experiment variability in disease incidence and severity, plants should be grown for 10 days before inoculation in a well-controlled growth chamber. 
Despite inter-experiment variability, overall disease severity increased with the number of infection sites on cotyledons. This agrees with the dose-response relationship established by Hammond \& Lewis (1987), i.e. with increasing inoculum spore concentration, the incidence of phoma leaf spots increased and an increasing proportion of phoma leaf spots generated more systemic biotrophic growth. The contrasted inoculation methods employed, i.e. different infection sites vs. one infection site with different spore concentrations at inoculation, have contrasted implications for field disease epidemiology. Results here suggest that on a given plant, high numbers of phoma leaf spots caused either by high ascospore showers or by secondary cycles implying conidia rain-splashed over short distances, could facilitate the establishment of the systemic biotrophic growth of the fungus. Assuming that the abscission of an infected leaf occurs after the completion of fungal systemic growth into the petiole (Hammond \& Lewis, 1986), this could lead to severe stem canker epidemics.

The differential reaction between the susceptible and resistant lines (Table 1) indicates that the method developed here could be used for screening for quantitative resistance, while its potential use to differentiate more levels of host quantitative resistance remains to be tested. Evaluating levels of quantitative resistance in B. napus requires controlled conditions to eliminate the host genotype $\times$ environment interaction occurring in the field (Newman \& Bailey, 1987; Bansal et al., 1994). Disease incidence and severity on plants with polygenic resistance were significantly decreased compared to susceptible plants, confirming previous field studies (Delourme et al., 2006). This result emphasizes the potential effectiveness of polygenic resistance in managing phoma stem canker. Polygenic resistant cultivars do not affect leaf infections and are assumed to react equally across all $L$. maculans strains during stem colonization, but this type of resistance might exert a selection pressure on the pathogen population and promote the most aggressive strains, resulting in increased aggressiveness at the population level. The method described could also be used to characterize pathogen aggressiveness (expressed both as the speed of stem colonization and severity of the resulting disease). Even if the two isolates were only slightly different in their ability to reach the hypocotyl (Fig. 4), field studies suggested that virulent avrLm4 isolates are less competitive than avirulent isolates during growth down the petiole to reach the stem base of susceptible hosts (Huang et al., 2006). A season-to-season pathogen population field survey on polygenic resistant cultivars combined with the experimental method proposed in this paper could assess the evolution of population aggressiveness.

In the course of systemic growth, competition and colonization efficiency may determine which individuals will reach the site of sexual reproduction. Hypocotyl colonization efficiency of both studied isolates decreased when the number of infection sites of the competing isolate on cotyledons was increased (Fig. 4). Schmit et al. (2002) showed that only a few L. maculans strains isolated from phoma leaf spots in the autumn could be reisolated from the basal stem canker before harvest, which can be explained by competition between fungal strains during systemic growth. Competition for resources between $L$. maculans and $L$. biglobosa, the two related species causing blackleg (Shoemaker \& Brun, 2001), was suggested during the saprophytic stage on oilseed rape stubble (West et al., 2002). To better understand competition during systemic growth, which could be caused by either spatial exclusion among hyphae or trophic competition at any step of the colonization (in lamina, petiole or stem), the use of fluorescent protein-expressing strains would be very helpful.

Apart from the direct consequences of disease development on yield, the systemic progress of $L$. maculans is a key stage for the fungus to access the site of sexual reproduction. The present results from controlled conditions suggest that at low L. maculans population densities, access to the site of sexual reproduction may depend on the number of phoma leaf spots per plant during the early susceptible stage. Hence, the occurrence of early secondary cycles, generating clusters of secondary phoma leaf spots on the same plant, should promote systemic growth and therefore sexual reproduction at the stem base. In addition, competitive effects might affect strains during systemic growth, with a density-dependent relative colonization success, as reported here. Results confirmed that cultivars with polygenic resistance reduce disease severity; a further step would be to study if the ability of isolates to reach the stem and to mate sexually is subsequently also reduced. 


\section{Acknowledgements}

This research is part of a $\mathrm{PhD}$ project funded jointly by Institut National de la Recherche Agronomique (INRA) and Région Bretagne. This work was carried out with the financial support of the Ministère de l'Agriculture et de la Pêche (CTPS 05-02 Phoma). The authors thank H. Miteul for technical assistance.

\section{References}

Ansan-Melayah D, Balesdent MH, Buée M, Rouxel T, 1995. Genetic characterization of AvrLml, the first avirulence gene of Leptosphaeria maculans. Phytopathology 85, 1525-9.

Ansan-Melayah D, Balesdent MH, Delourme R et al ., 1998. Genes for race-specific resistance against blackleg disease in Brassica napus L. Plant Breeding 117, 373-8.

Aubertot JN, Sohbi Y, Brun H, Penaud A, Nutter FW, 2006a. Phomadidacte: a computer-aided training program for the severity assessment of phoma stem canker of oilseed rape. IOBC Bulletin 29, 24754.

Aubertot JN, West JS, Bousset-Vaslin L, Salam MU, Barbetti MJ, Diggle AJ, 2006b. Improved resistance management for durable disease control: a case study of phoma stem canker of oilseed rape (Brassica napus). European Journal of Plant Pathology 114, 91-106.

Baird RE, Phillips DV, Mullinix G, Alt PJ, 1999. Relative longevity of Leptosphaeria maculans and associated mycobiota on canola debris. Phytoprotection 80, 1-11.

Bansal VK, Kharbanda PD, Stringam GR, Thiagarajah MR, Tewari JP, 1994. A comparison of greenhouse and field screening methods for blackleg resistance in doubled haploid lines of Brassica napus. Plant Disease 78, 276-81.

Begon M, Townsend CR, Harper JL, 2006. Ecology: From Individuals to Ecosystems, 4th edn. Oxford, UK: Blackwell Publishing.

Brun H, Levivier S, Ruer D, Somda I, Chèvre AM, Renard M, 2000. A field method for evaluating the potential durability of new resistance sources: application to the Leptosphaeria maculans/Brassica napus pathosystem. Phytopathology 90, 961-6.

Brunin B, Lacoste L, 1970. Recherches sur la maladie du colza due àLeptosphaeria maculans (Desm.) Ces. et de Not. II. Pouvoir pathogène des ascospores. Annales de Phytopathologie 2, 477-88.

De March G, Séguin-Swartz G, Petrie GA, 1986. Virulence and culture filtrate phytotoxicity in Leptosphaeria maculans: perspectives for in vitro selection. Canadian Journal of Plant Pathology $8,422-8$.

Delourme R, Chèvre AM, Brun H et al ., 2006. Major gene and polygenic resistance to Leptosphaeria maculans in oilseed rape (Brassica napus). European Journal of Plant Pathology 114, 41-52.

Fitt BDL, Brun H, Barbetti MJ, Rimmer SR, 2006. World-wide importance of phoma stem canker (Leptosphaeria maculans and L. biglobosa) on oilseed rape (Brassica napus). European Journal of Plant Pathology 114, 3-15.

Hall R, 1992. Epidemiology of blackleg of oilseed rape. Canadian Journal of Plant Pathology 14, 46-55.

Hammond KE, Lewis BG, 1986. The timing and sequence of events leading to stem canker disease in populations of Brassica napus var. oleifera in the field. Plant Pathology 35, 551-64.

Hammond KE, Lewis BG, 1987. The establishment of systemic infection in leaves of oilseed rape by Leptosphaeria maculans. Plant Pathology 36, 135-47.

Hammond KE, Lewis BG, Musa TM, 1985. A systemic pathway in the infection of oilseed rape plants by Leptosphaeria maculans. Plant Pathology 34, 557-65.

Huang YJ, Li Z-Q, Evans N, Rouxel T, Fitt BDL, Balesdent MH, 2006. Fitness cost associated with loss of the AvrLm4 avirulence function in Leptosphaeria maculans (phoma stem canker of oilseed rape). European Journal of Plant Pathology 114, 77-89.

Huang YJ, Pirie E, Evans N, Delourme R, King GJ, Fitt BDL, 2007. Understanding symptomless growth of Leptosphaeria maculans in Brassica napus (oilseed rape) to manage phoma stem canker. In: Proceedings of the 12th International Rapeseed Congress, Wuhan, China, March 26-30 vol. IV, 146.

Huang YJ, Pirie E, Evans N, Delourme R, King GJ, Fitt BDL, 2009. Quantitative resistance to symptomless growth of Leptosphaeria maculans (phoma stem canker) in Brassica napus (oilseed rape). Plant Pathology 58, 314-23. 
Huber L, Gillespie TJ, 1992. Modeling leaf wetness in relation to plant disease epidemiology. Annual Review of Phytopathology 30, 533-77.

Johnson RD, Lewis BG, 1994. Variation in host range, systemic infection and epidemiology of Leptosphaeria maculans. Plant Pathology 43, 269-77.

Kutcher HR, Van Den Berg CGJ, Rimmer SR, 1993. Variation in pathogenicity of Leptosphaeria maculans on Brassica spp. based on cotyledon and stem reactions. Canadian Journal of Plant Pathology 15, 253-8.

Li H, Sivasithamparam K, 2003. Breakdown of a Brassica rapa subsp. sylvestris single dominant blackleg resistance gene in B. napus rapeseed by Leptosphaeria maculans field isolates in Australia. Plant Disease 87, 752.

McGee DC, 1977. Black leg (Leptosphaeria maculans (Desm.) Ces. et de Not.) of rapeseed in Victoria: sources of infection and relationships between inoculum, environmental factors and disease severity. Australian Journal of Agricultural Research 28, 53-62.

Nathaniels NQR, Taylor GS, 1983. Latent infection of winter oilseed rape by Leptosphaeria maculans. Plant Pathology 32, 23-31.

Ndimande B, 1976. Studies on Phoma lingam (Tode ex Fr.) Desm. and the Dry Rot of Oil Seed Rape, Brassica napus (L.) var. oleifera Metzger. Uppsala, Sweden: Agricultural College of Sweden, PhD thesis.

Newman PL, 1984. Differential host-parasite interactions between oilseed rape and Leptosphaeria maculans, the causal fungus of stem canker. Plant Pathology 33, 205-10.

Newman PL, Bailey DJ, 1987. Screening for resistance to canker (Leptosphaeria maculans) in winter oilseed rape (Brassica napus ssp. oleifera). Plant Pathology 36, 346-54.

Newton MR, Kinkel LL, Leonard KJ, 1997. Competition and density-dependent fitness in a plant parasitic fungus. Ecology 78, 1774-84.

Petrie GA, Lewis PA, 1985. Sexual compatibility of isolates of the rapeseed blackleg fungus Leptosphaeria maculans from Canada, Australia, and England. Canadian Journal of Plant Pathology 7, 253-5.

Pilet ML, Delourme R, Foisset N, Renard M, 1998. Identification of QTL involved in field resistance to light leaf spot (Pyrenopeziza brassicae) and blackleg resistance (Leptosphaeria maculans) in winter rapeseed (Brassica napus L.). Theoretical and Applied Genetics 97, 398-406.

Pringle A, Taylor JW, 2002. The fitness of filamentous fungi. Trends in Microbiology 10, 474-81.

Roussel S, 1999. Expression de la Résistance du Colza àLeptosphaeria maculans au Stade Jeune et au Stade Adulte: Caractérisation Cytologique et Epidémiologique. Rennes, France: University of Rennes $1, \mathrm{PhD}$ thesis.

Rouxel T, Penaud A, Pinochet X et al ., 2003. A 10-year survey of populations of Leptosphaeria maculans in France indicates a rapid adaptation towards the Rlm1 resistance gene of oilseed rape. European Journal of Plant Pathology 109, 871-81.

SAS, 2000. Statistical Analysis System, SAS/STAT User's Guide, Version 8.1. Cary, NC, USA: SAS Institute Inc.

Schmit J, West J, Narcy JP et al ., 2002. La nécrose du collet du colza : analyse de la distribution du champignon dans la plante à l'aide d'outils moléculaires. Oléagineux Corps gras Lipides 9, 2-3.

Shoemaker RA, Brun H, 2001. The teleomorph of the weakly aggressive isolates segregate of Leptosphaeria maculans. Canadian Journal of Botany 79, 412 -9.

Smith HC, Sutton BC, 1964. Leptosphaeria maculans the ascogenous state of Phoma lingam. Transactions of the British Mycological Society 47, 159-65.

Somda I, Delourme R, Renard M, Brun H, 1999. Pathogenicity of Leptosphaeria maculans isolates on a Brassica napus-B. juncea recombinant line. Phytopathology 89, 169-75.

Sprague SJ, Balesdent MH, Brun H et al ., 2006. Major gene resistance in Brassica napus (oilseed rape) is overcome by changes in virulence of populations of Leptosphaeria maculans in France and Australia. European Journal of Plant Pathology 114, 33-40.

Travadon R, Bousset L, Saint-Jean S, Brun H, Sache I, 2007. Splash dispersal of Leptosphaeria maculans pycnidiospores and the spread of blackleg on oilseed rape. Plant Pathology 56, 595-603. 
West J, Balesdent MH, Rouxel T et al ., 2002. Colonization of winter oilseed rape tissues by A/Tox ${ }^{+}$and B/Tox ${ }^{0}$ Leptosphaeria maculans (phoma stem canker) in France and England. Plant Pathology 51, 311-21.

West JS, Kharbanda PD, Barbetti MJ, Fitt BDL, 2001. Epidemiology and management of Leptosphaeria maculans (phoma stem canker) on oilseed rape in Australia, Canada and Europe. Plant Pathology $50,10-27$.

Xi K, Morrall RAA, Gugel RK, Verma PR, 1991. Latent infection in relation to the epidemiology of blackleg of spring rapeseed. Canadian Journal of Plant Pathology 13, 321-31. 